\title{
Adaptive calibration for small parallel coordinate measuring machine
}

\author{
Ryoshu Furutani \\ Department of Advanced Machinery Engineering, School of Engineering, Tokyo Denki University
}

Correspondence Author: Ryoshu Furutani, Department of Advanced Machinery Engineering, School of Engineering, Tokyo Denki University. E-mail: - ryo@mail.dendai.ac.jp

Received date: 20 May 2019, Accepted date: 19 June 2019, Online date: 28 July 2019

Copyright: () 2019 Ryoshu Furutani, et al., This is an open-access article distributed under the terms of the Creative Commons Attribution License, which permits unrestricted use, distribution, and reproduction in any medium, provided the original author and source are credited.

\begin{abstract}
The small parallel coordinate measuring machine had been developed to measure a small workpiece with high accuracy. The small parallel CMM in this paper has the structure that the platform has three rotational joints supported by PZT actuators respectively. Until now, the platform of CMM was assumed rigid and the kinematic parameters were calibrated. As the calibration result was insufficient in the rigid model, the deformation of the rotational joints was introduced. However, this model did not accomplish sufficient calibration. In this paper, the deep learning was introduced to express the relationship between the extensions of PZTs and the coordinates of CMM. At first, the structure and the model of small parallel CMM were shown. The calibration results based on the rigid model and the deep learning were shown and compared.
\end{abstract}

Keywords: Kinematic parameters, kinematic calibration, neural network, adaptive calibration

\section{INTRODUCTION}

The mechanism with two or more degree of freedom is referred to as a mechanism with multi-degree-of-freedom. They are categorized into two types. One is the parallel mechanism of which the actuators are disposed of in parallel and the other is the serial mechanism of which the actuators are disposed of in series. The serial mechanism has been used in many industrial robots because it can take the wider attitude angle and work area (Takeda 2005). However, it is known that the serial mechanism has the problem that the errors are accumulated. On the other hand, as the actuators in the parallel mechanism are arranged in parallel, it is considered to be more accurate than a serial mechanism. Therefore, it is expected to be used in a precision positioning device (Schwenke, 2008; Agaplou, 2007).

However, the kinematic parameters of the parallel mechanism have a large correlation with the other parameters (Takamasu 2003; Torii 2010; Chiu 2003). So, in the self-calibration method based on the kinematic equations, the kinematic parameters were not estimated well enough (Ohnishi, 2007; Yokouchi 2014; Takamasu, 2004; Sato, 2004). Women.

\section{KINEMATIC CALIBRATION BASE ON LEAST SQUARED METHOD MATERIAL AND METHODS}

The calibration process of the coordinate measuring machines is shown in Figure 1 (a). The actual CMM measures the coordinates of the feature of the artifact, and the sensor information is detected by the actual CMM. The coordinates are temporally calculated using the model of the coordinate measuring machine and the estimated kinematic parameters. In the estimator, the temporally calculated coordinates are compared with the calibrated coordinates and the kinematic parameters are estimated. These estimated kinematic parameters are used with the model of the coordinate measuring machine, as shown in Figure 1 (b).

The measurement process is shown in Figure 1 (b). The coordinate measuring machines are used to measure the coordinates of the workpiece. In the measurement process, the actual CMM measures the coordinates of the feature of the workpiece and the sensor information is detected by the actual CMM. The coordinates are calculated using the model of the coordinate measuring machine and the estimated kinematic parameters. 
It is necessary that the model and the kinematic parameters are separated and defined in the measurement system based on the usual calibration using the artifact, as shown in Figure 1.

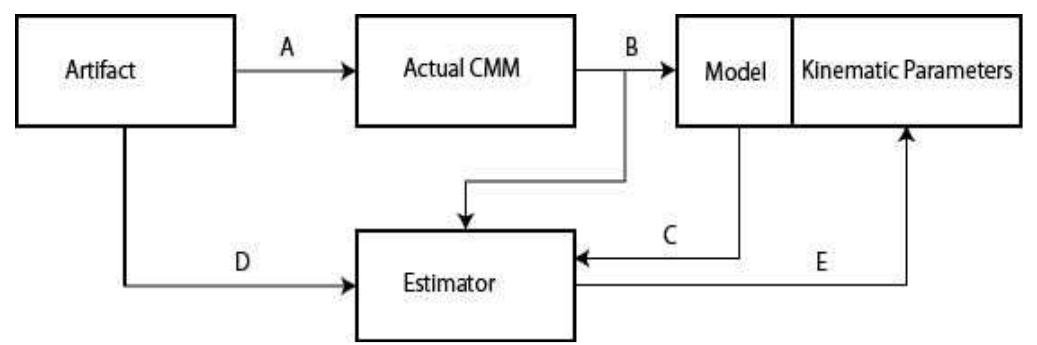
A: feature of the artifact,
B: sensor information
C: calculated coordinates
D: calibrated coordinates
E: estimated kinematic parameters

(a) Diagram of estimation of kinematic parameters using the artefact

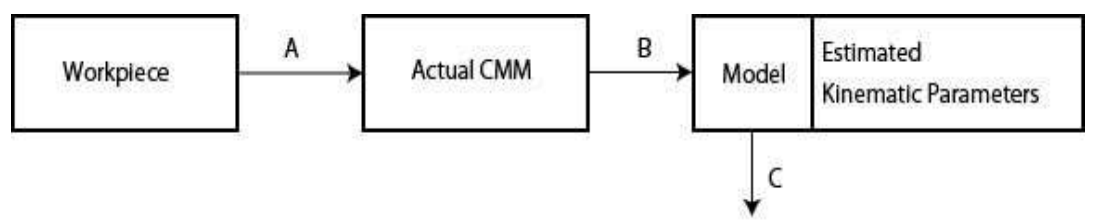

A: feature of the workpiece
B: sensor information
C: calculated coordinates

(b) Diagram of measurement using estimated kinematic parameters

Figure 1: Usual calibration and measurement

\section{ADAPTIVE CALIBRATION BASE ON DEEP LEARNING}

In the calibration, the model equations, the kinematic parameters and the estimators, as shown in Figure 1(a) are replaced with the neural network, as shown in Figure 2(a). The calibration method using the neural network is feasible and flexible. So it is called the adaptive calibration.

The neural network learns the relationship between the sensor information and the calibrated coordinates of the artifact. The neural network could express the equation, including the deformation of the stage and the arms in the case of the parallel coordinate measuring machine.

In measurement, the model equation and the kinematic parameters, as shown in Figure 1(b) are also replace with the neural network, as shown in Figure 2(b). In Figure 2, the neural network studies in the adaptive calibration, and it does not study in the measurement.

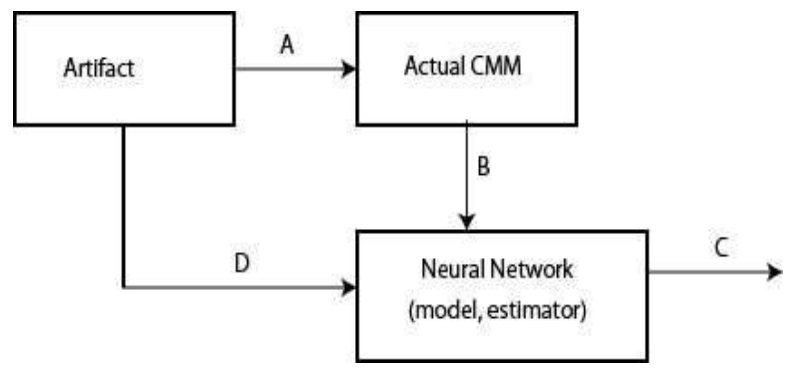
A: feature of artifact
B: sensor information
C: calculated coordinates
D: calibrated coordinates

(a) Kinematic parameters are estimated adaptively using the artifact

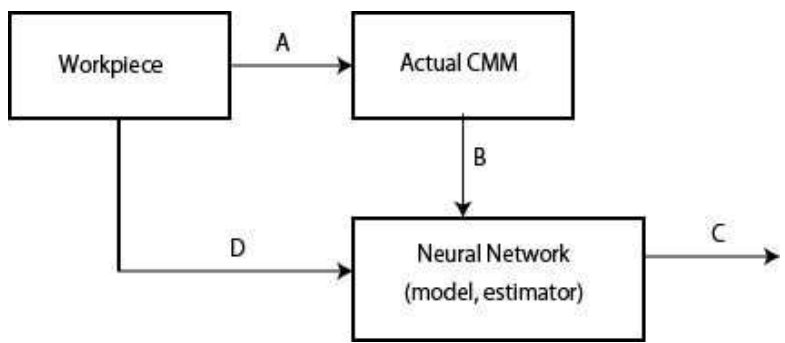

A: feature of the workpiece

B: sensor information

C: calculated coordinates

(b) Coordinates are calculated using adaptively estimated kinematic parameters.

Figure 2: Adaptive calibration and measurement

\section{MODEL OF PARALLEL MECHANISM AND KINEMATIC PARAMETERS}

The model of the parallel mechanism of this research is shown in Figure 3. This parallel mechanism has three actuators and the end effector is supported at the rotational joints of the elastic hinges by three actuators. 
The definition of the kinematic parameters is shown here. (wx1, wy1), (wx2, wy2) and (wx3, wy3) is one end of the actuator to the base plate and are represented by the world coordinate system. (px, py) is the stylus coordinates of the triangle stage and is represented by the world coordinate system. ( $\Delta \mathrm{px}, \Delta \mathrm{py})$ is the displacement of the stylus. (sx1, sy1), (sx2, sy2) and (sx3, sy3) are the coordinates of the vertex of the triangle stage and are represented by a local coordinate system with the origin of the stylus location. L1, L2 and L3 are actuator length. $\Delta \mathrm{L} 1, \Delta \mathrm{L} 2$ and $\Delta \mathrm{L} 3$ are actuator amount of expansion and contraction. $\theta$ is the rotation angle and is positive when the stage rotates in clockwise.

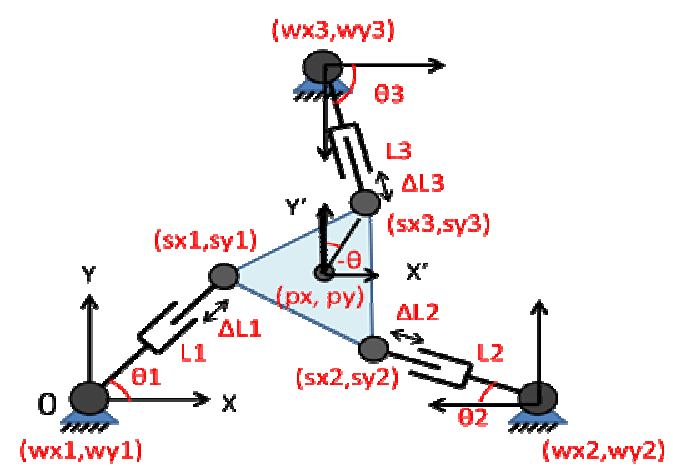

Figure 3: Model of the 2D planar coordinate measuring machine

\section{D PARALLEL MECHANISM WITH THREE DEGREE OF FREEDOM}

The 2D parallel mechanism is shown in Figure 4(a). Three bases are fixed on the world coordinate system. Three actuators are disposed between the base and the stage. The stage is supported by three actuators and can move in $\mathrm{x}$ and $\mathrm{y}$ direction and rotation according to the variations of three actuator lengths. The $2 \mathrm{D}$ parallel mechanism is fixed on the base plate as shown in Figure 4(b) and the coordinate system of this device is related with the world coordinate system, which is sometimes called the coordinate machine system.

The used actuators are a laminated piezoelectric element (NEC Tokin, AE0505D16F) of which the maximum amount of displacement is $17.4 \pm 2.0[\mu \mathrm{m}]$ and generating force is $850[\mathrm{~N}]$ at the time of applying DC150V. Also, the amount of displacement of the stacked piezoelectric element is up to $17 \mu \mathrm{m}$. The rotation angle of stage and the amount of displacement of the stage are measured at the stylus point by the capacitive displacement gauge (Iwatsu Electric Co., Ltd., ST-3511, ST-0532A) as shown in Figure 4(b).

The stage rotation angle is calculated from the difference between the stage displacement of the coaxial direction of displacement and rotation angle observation auxiliary plate as shown in Figure 4(b), and the distance between the axes of two capacitive displacement gauges.

Figure 5 shows the situation that the parallel mechanism is set up and the measuring gauges are set up for experiments.

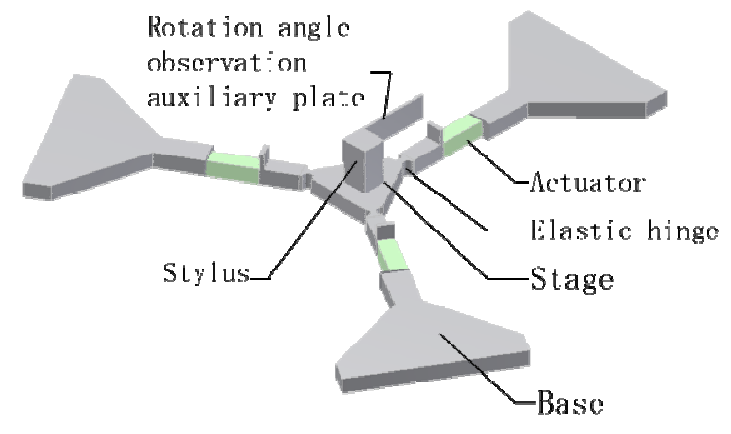

(a) The 2D parallel mechanism consists of the bases, actuators and stage. The stage is supported by three actuators.

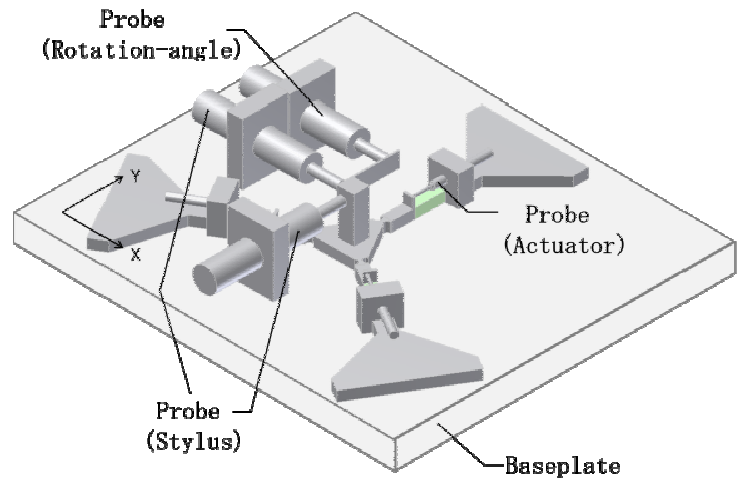

(b) The $2 \mathrm{D}$ parallel mechanism is set on the baseplate. Six measuring instruments are set up.

Figure 4: 2D parallel mechanism and measuring instruments for calibration 


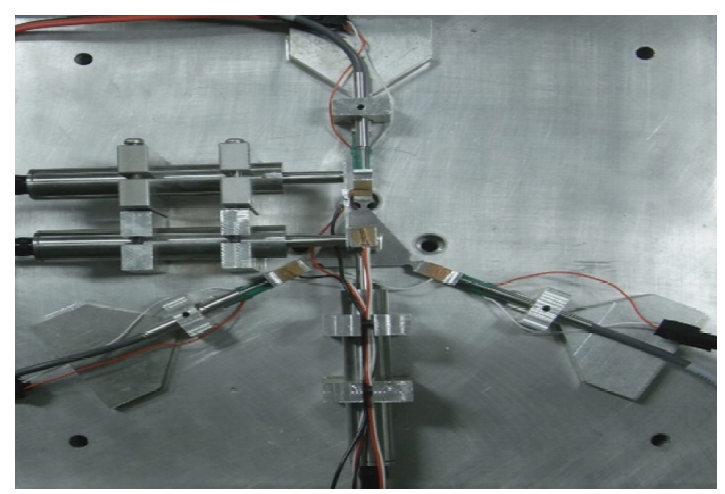

Figure 5: Picture of the parallel mechanism and measuring gauges.

\section{EXPERIMENT}

In this paper, the fully-connected neural network with 5 layers is adapted. The node of the input layer is 3 for the actuator lengths and the node of the output layer is 2 for the $\mathrm{x}$ and $\mathrm{y}$ location.

In an experiment, the piezo actuators are applied with every $15 \mathrm{~V}$ from 0 to $150 \mathrm{~V}$. So, the stylus locations, i.e., $\mathrm{x}$ and $\mathrm{y}$ coordinates, and the lengths of three piezo actuators are measured at $1331(=11 \times 11 \times 11)$ points. Both calibration methods apply to these data. The kinematic parameters, as shown in Figure 3, are estimated by Least Squares Method and the neural network is trained using a part of these data.

Figure 6(a) shows the deviation in $\mathrm{x}$ coordinate after kinematic calibration and Figure 6(b) shows the deviation in $\mathrm{x}$ coordinate, which is estimated by the trained neural network.

It is proved that the neural network could replace the calibration of the kinematic parameters of measuring instruments.

\section{CONCLUSION}

It was proposed that the calibration process of the kinematic parameters of the parallel CMM could be replaced with the training of the neural network. The trained neural network was applied to the parallel CMM, and the calibration result was smaller than the least squared method. At this moment, the meta parameters, e.g., the number of hidden layers and the number of nodes in hidden layers, are not discussed. As these meta parameters are imported to estimate the behavior of measuring instrument, some parameters are necessary to be optimized.

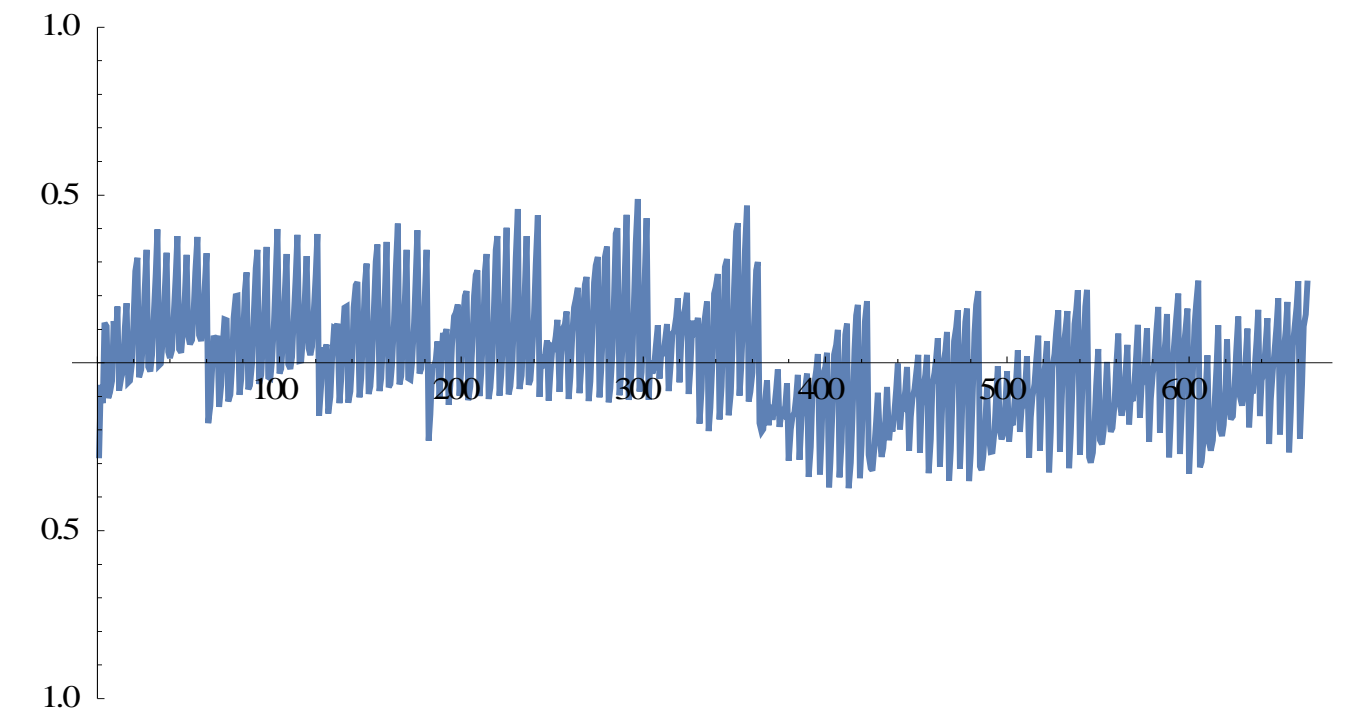

(a) The deviation in $\mathrm{x}$ coordinate after kinematic calibration 


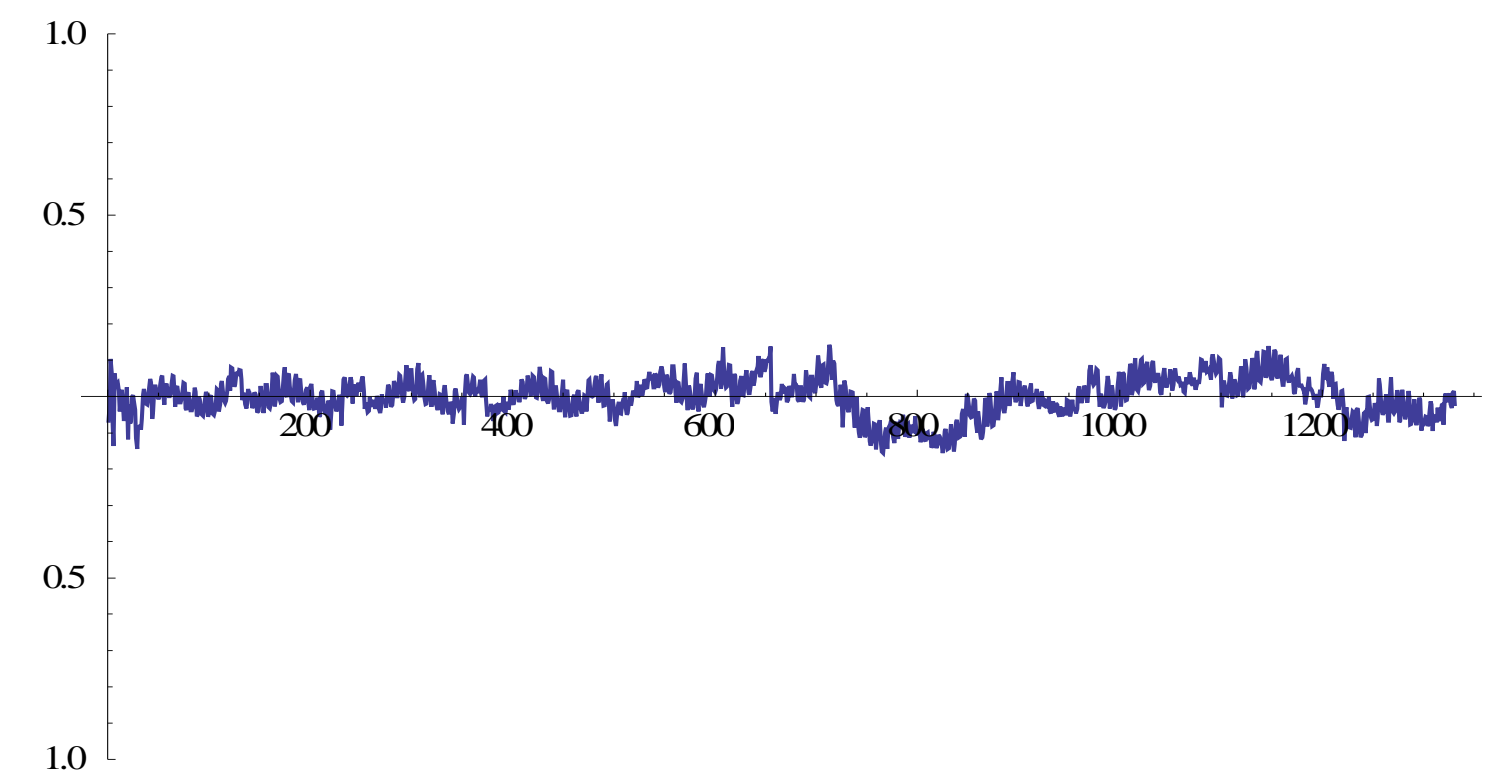

(b) The deviation in $\mathrm{x}$ coordinate which is estimated by the trained neural network

Figure 6: The deviation in $\mathrm{x}$ coordinates

\section{REFERENCES}

Agaplou,J.S. , Asuring,H.D, 2007. Assuring the Day to -Day Accuracy of Coordinate Measuring Machines - A Comparison of Tools and Procedures, Journal of Manufacturing Process, 9(2),109-120

Chiu,Y.J. and Perng,M.H., 2003. Self-calibration of a general hexapod manipulator using cylinder constraints, Machine Tools and Manufacture, 43,1051-1066

Ohnishi,T. and et al., 2007. Study of Improvement mechods of CMM (Coordinate Measuring Machine) in Workshop Environment - Correction of Squareness Error-, Journal of JSPE, 73(7), 818-822

Sato,O. and et al., 2004. Artefact calibration of parallel mechanism, kinematic calibration with a priori knowledge, Measurement Science and Technology, 15, 1158-1165

Schewenke,H. and et al.,2008. Geometric error measurement and compensation of machines - an update, CIRP AnnalsManufacturing Technology, 57, 660-675

Takamasu,K. and et al., 2003. Artifact calibration of coordinate measuring machine, Journal of JSPE, 69(6), 851-855

Takamasu,K. and et al., 2004. Artefact Calibration of Coordinate Measuring Machine (2nd Report)-Self-Calibration of Redundant Coordinate Machine-, Journal of JSPE, 70(5), 711-715

Takeda,Y, 2005. Parallel Mechanism (Introduction to Precision Engineering), J of JSPE ,71(11), 1363-1368 